\title{
CORRELATION OF SERUM OSTEOCALCIN LEVEL AND PERIODONTAL ATTACHMENT LOSS WITH OSTEOPOROSIS RISK STATUS IN POSTMENOPAUSAL WOMEN
}

\author{
DIMAS ILHAM HUTOMO ${ }^{1}$, SRI LELYATI C MASULILI ${ }^{1 *}$, FATIMAH MARIA TADJOEDIN ${ }^{1}$, LINDAWATI S KUSDHANY²
}

${ }^{1}$ Department of Periodontics, Faculty of Dentistry, Universitas Indonesia, Jakarta, Indonesia. ${ }^{2}$ Department of Prosthodontics, Faculty of Dentistry, Universitas Indonesia, Jakarta, Indonesia. Email: srilelyati@yahoo.com

Received: 16 September 2017, Received and Accepted: 03 October 2017

\section{ABSTRACT}

Objective: To assess the relationship between serum osteocalcin and periodontal clinical attachment loss (CAL) in postmenopausal women in relation to their osteoporosis risk status.

Methods: A cross-sectional study was carried out on 71 postmenopausal women in Kenari District, Central Jakarta, Indonesia. The periodontal examination for all the subjects included a CAL measurement. The serum osteocalcin level was analyzed using ELISA.

Results: The correlation between the serum osteocalcin level in patients with CAL and the risk of osteoporosis was analyzed with the Spearman test. The normal group had 29 subjects (40.84\%), the osteopenic group had 23 subjects (32.39\%), and the osteoporotic group had 19 subjects (26.76\%). There was a significant correlation between CAL and the osteoporosis high-risk status $(\mathrm{p}<0.05)$ and no significant correlation between the serum osteocalcin level and the osteoporosis status ( $\mathrm{p}>0.05)$.

Conclusion: No correlation was found between CAL and the serum osteocalcin level (p>0.05).

Keywords: Osteoporosis, Osteocalcin, Clinical attachment loss, Postmenopausal women.

(C) 2017 The Authors. Published by Innovare Academic Sciences Pvt Ltd. This is an open access article under the CC BY license (http://creativecommons. org/licenses/by/4. 0/) DOI: http://dx.doi.org/10.22159/ijap.2017.v9s2.22

\section{INTRODUCTION}

Osteoporosis is defined as a bone disease characterized by a decrease in bone mass and alterations in microarchitecture that result in bone fragility and an increased risk of fractures in the future [1]. Osteoporosis results from an imbalance between the rates of bone formation and resorption, which leads to the loss of bone mineral mass [2]. Based on data from the international osteoporosis foundation in 2013, the prevalence of osteoporosis in Indonesian women aged 50-80 years old is $23 \%$ and in those aged $70-80$ years is $53 \%$. By 2050 , one-third of the total population will be at high risk for osteoporosis [3].

Periodontitis is a destructive inflammatory disease of the toothsupporting structures and is a major cause of tooth loss. Its presence is determined by the measurement of clinical attachment loss (CAL) [4]. The etiology of this disease is periodontal pathogens found in dental plaque that results in the inflammation of the tooth-supporting structure [2]. Periodontal diseases are correlated with several systemic diseases such as cardiovascular diseases, diabetes mellitus, osteoporosis, respiratory diseases, and others [5].

Both osteoporosis and periodontitis are bone-destroying diseases. They are characterized by increased bone resorption, host dependence, a multifactorial etiology, and stimulated by cytokine activity [4]. The relationship between osteoporosis and periodontal diseases is one of the bidirectional interferences. The reduction in bone mineral density in osteoporosis alters the trabecular pattern and may lead to a more rapid jawbone resorption caused by periodontal disease, due to the invasion of periodontal pathogenic bacteria. Conversely, the invasion of periodontal pathogenic bacteria may alter the normal homeostasis of the alveolar bone, increasing the osteoclastic activity and reducing the local and systemic bone density [6]. Pepelasi et al. [7] suggested that postmenopausal women with osteoporosis had greater gingival inflammation, periodontal attachment loss, and gingival recession than postmenopausal women with a normal bone mineral density (BMD).

The American Academy of Periodontology suggested that osteoporosis is not an etiology for periodontal disease but that it can exaggerate alveolar bone destruction [8]. Postmenopausal women with a reduced BMD have a greater tendency for alveolar bone loss and CAL because inflammatory mediators are increased in the systemic and oral bones [9]. Bone turnover can be assessed by measurements of a protein produced by osteoblasts and osteoclasts: Osteocalcin, also known as the bone Gla protein, which is a noncollagenous bone protein [10].

Noncollagenous bone matrix proteins play a key role in matrix mineralization, cellular adhesion, and the regulation of cell activity during the coupling of bone formation and resorption. Osteocalcin, one of the most abundant of these proteins, plays a key role in mineralization, may act as a chemoattractant, and may be essential for osteoclast differentiation [11]. It is synthesized mainly by osteoblasts as well as a small number of odontoblasts and chondroblasts. After being synthesized, it is stored in the bone mineral matrix as hydroxyapatite crystals and is recognized as a marker of bone formation [10]. Serum osteocalcin is known to be a valid biomarker for detecting a low BMD [12].

Increases in serum osteocalcin levels are associated with rapid bone loss. In osteoporosis, there is a deficiency of the calcium level and since osteocalcin is known as a calcium-dependent biomarker and has a strong affinity with hydroxyapatite responsible for bone mineralization. Osteoporosis leads to decreased hydroxyapatite crystal formation, and hence, results in an increase in serum osteocalcin levels [13].

To the best of our knowledge, there have been no studies analyzing the association between serum osteocalcin and periodontal status in 
postmenopausal women with osteoporosis in Indonesia. The objective of this study was to assess the relationship of serum osteocalcin and periodontal CAL levels with the risk of osteoporosis in postmenopausal women.

\section{METHODS}

A cross-sectional study was carried out in 71 postmenopausal women ranging in age from 48 to 87 years in the Kenari District, Central Jakarta, Indonesia. The inclusion criteria were women who had already had menopause for at least 1 year from their last menstrual period, who still had natural teeth in their maxilla and mandible, who were able to communicate, and who were willing to participate in this study. The exclusion criteria were women with systemic diseases, who were former smokers and alcoholics, who had received hormone therapy within the past 5 years, and who had had a hysterectomy. This study was approved by the Ethical Committee of the Faculty of Dentistry, Universitas Indonesia. All of the subjects signed informed consent forms before joining this study.

The risk of osteoporosis was determined by the mandibular bone density index. This index determines the risk of osteoporosis in subjects by assessing their age, duration of menopause, body mass index, history of hormonal therapy, duration of daily sun exposure, daily activity, and calcium intake. The subjects were classified into three groups: Osteoporosis (high risk), osteopenia (medium risk), and normal (low or no risk). A score from -9 to 25 meant osteoporosis, 26-52 meant osteopenia, and 53-95 was normal [14].

A periodontal examination was carried out for all subjects, and the following measurements were recorded to determine the level of CAL. CAL was measured from the cement-enamel junction to the base of the pocket using a periodontal probe. All of the measurements were assessed at six sites per tooth: Mesiobuccal, midbuccal, distobuccal, mesiolingual, midlingual, and distolingual.

A syringe was used to draw $5 \mathrm{~mL}$ of venous blood, which was then centrifuged for $10 \mathrm{~min}$. The samples were stored in small capped vials for long-term use at $-20^{\circ} \mathrm{C}$ until tested. The serum was examined by the ELISA method, performed at Laboratorium Terpadu, Faculty of Medicine, Universitas Indonesia, Jakarta.

The data were analyzed using SPSS software from IBM. A normality test was performed. The correlations of age, serum osteocalcin level, and CAL with the osteoporosis risk were tested using a Spearman correlation test. Only $\mathrm{p}<0.05$ was considered to be statistically significant, and a positive value showed a positive correlation. Comparison of serum osteocalcin levels with severity of CAL was tested using Kruskal-Wallis test.

\section{RESULTS}

Seventy-one postmenopausal women ranging in age from 48 to 87 years participated in this study. The subjects were classified into three groups: Normal, with 29 subjects (40.28\%); osteopenia, with 23 subjects (31.94\%); and osteoporosis, with 19 subjects (26.39\%). The mean values of the serum osteocalcin level and CAL for all three groups are presented in Table 1 . The osteoporosis group had a higher mean age, mean serum osteocalcin level, and mean CAL than the other groups.
To investigate the correlations between the serum osteocalcin and CAL levels and the osteoporosis risk, the data were analyzed with a Spearman correlation test. Table 2 summarizes that there was a positive correlation between CAL and the osteoporosis high-risk status ( $\mathrm{r}=0.32$; $\mathrm{p}<0.05$ ), but there was no correlation between serum osteocalcin levels and the osteoporosis risk $(\mathrm{p}>0.05)$.

Table 3 summarizes that there was no difference in the osteocalcin levels among subjects with mild, moderate, and severe CAL. The serum osteocalcin level was higher in those with mild and moderate CAL than with severe CAL. These results suggest that there is no statistically significant difference in the serum osteocalcin levels of the subjects with mild, moderate, and severe CAL ( $p>0.05$ ).

\section{DISCUSSION}

This study was conducted in postmenopausal women because there is a strong relationship between menopause and osteoporosis. In postmenopausal women, hormonal changes cause an increase in receptor activator of nuclear factor Kappa-B ligand (RANKL), as does osteoclast activity. As a consequence, there is a shift from bone remodeling toward bone resorption that leads to osteoporosis [15].

The World Health Organization has determined that dual-energy X-ray absorptiometry is the gold standard for diagnosing osteoporosis. In this study, we used a questionnaire that determined the subjects' risk factors for osteoporosis (the mandibular bone density index) [14].This method was used because the index was developed based on a scoring model that consists of risk factors for osteoporosis. The index has a sensitivity of $77.06 \%$ and a specificity of $78.45 \%$ [14].

In individuals with osteoporosis, a low BMD in the jawbones may be associated with low systemic bone density. This low bone density or loss of bone density may lead to an increased susceptibility to the resorption of alveolar bone in areas with periodontitis. Systemic factors also modify the response of local tissue to periodontal infection and may also affect bone remodeling. Individuals with systemic bone loss have an increased systemic production of proinflammatory cytokines that may affect bone, including the maxilla and mandible. The result is a low bone density in the jawbones, caused by the increase in alveolar porosity, and more rapid alveolar bone resorption following an invasion by periodontal pathogens [16].

Bone remodeling occurs throughout the human lifespan. Bone is renewed by the bone turnover process, the products of which are known as bone turnover biomarkers and are classified as bone formation biomarkers and bone resorption biomarkers. Osteoblasts produce osteocalcin, which is important for calcification [17]. Serum osteocalcin is presently considered to be a valid marker of bone turnover when resorption and formation are coupled and a specific marker of bone formation when formation and resorption are uncoupled. Osteocalcin may be involved in recruiting osteoclasts to the sites of newly formed bone and thus may function as a negative regulator [18]

Table 1 summarizes that there is a tendency for serum osteocalcin levels and CAL to be higher in postmenopausal women with a high risk of osteoporosis than in the normal and osteopenia groups. Elevated serum osteocalcin levels have been found during periods of rapid bone turnover, as seen in osteoporosis, multiple myeloma,

Table 1: Characteristics of the three groups of subjects

\begin{tabular}{llll}
\hline Postmenopausal woman & \multicolumn{2}{l}{ Mean (SD) } & Osteoporosis (n=19) \\
\cline { 2 - 4 } & Normal (n=29) & Osteopenia (n=23) & $66(6.81)$ \\
\hline Age (years) & $56.55(7.86)$ & $62.26(6.34)$ & $1.03(1.32)$ \\
Serum osteocalcin (ng/mL) & $0.87(0.94)$ & $0.86(1.23)$ & $3.38(1.32)$ \\
CAL (mm) & $2.56(0.81)$ & $3.28(0.94)$ & \\
\hline
\end{tabular}

SD: Standard deviation, CAL: Clinical attachment loss 
Table 2: Correlations of serum osteocalcin and CAL levels with osteoporosis risk status

\begin{tabular}{llll}
\hline & \multicolumn{2}{l}{ Osteoporosis risk status } \\
\cline { 2 - 4 } & $\mathbf{n}$ & $\mathbf{r}$ & $\mathbf{p}$ \\
\hline Age & 71 & $0.56^{*}$ & $0.001^{*}$ \\
Serum osteocalcin & & 0.01 & 0.92 \\
CAL & & $0.32^{*}$ & $0.01^{*}$ \\
\hline
\end{tabular}

Spearman test, ${ }^{*} \mathrm{p}<0.05$ significance. CAL: Clinical attachment loss

Table 3: Comparison of serum osteocalcin levels in subjects with different severities of CAL

\begin{tabular}{lll}
\hline Severity of CAL & Osteocalcin $(\mathbf{n g} / \mathbf{m L})$ mean (SD) & p \\
\hline Mild $(1-2 \mathrm{~mm})$ & $0.63(0.45)$ & 0.31 \\
Moderate $(3-4 \mathrm{~mm})$ & $1.33(1.63)$ & \\
Severe $(\geq 5 \mathrm{~mm})$ & $0.61(0.37)$ & \\
\hline
\end{tabular}

Kruskal-Wallis test, $\mathrm{p}<0.05$ significance. CAL: Clinical attachment loss

and fracture repair [13]. Osteocalcin can be used as a marker of bone formation to give an idea about the change in bone turnover when it is normalized with respect to resorption [19]. Osteocalcin plays a role in bone resorption and the mineralization process, and it is involved in osteoclast recruitment to the sites of bone resorption and formation so that it may function as negative regulator [18].

The relationship between periodontal disease and osteoporosis is still controversial, but in this study, we suggest that there is a relationship between these diseases. Table 2 summarizes that CAL was positively correlated with osteoporosis high-risk status ( $\mathrm{p}=0.01, \mathrm{r}=0.32$ ), meaning that osteoporosis subjects had greater tendency toward higher CAL than the osteopenia or normal subjects. This finding is similar to that of a study conducted by Pepelassi et al., [7] which showed that osteoporotic women had significantly greater CAL, and severe periodontitis was most frequent among osteoporotic women. A similar result was reported in a systematic review by Dodd et al. [9] of the five longitudinal studies reviewed, four showed an association between osteoporosis and periodontal disease, 20 of 25 cross-sectional studies demonstrated a relationship between the two diseases, and all three case-control studies also showed an association. In contrast to this study, Masulili et al. [20] showed that there was no significant relationship between CAL and BMD.

Table 3 summarizes that there was no statistically significant association between the serum osteocalcin levels and the severity of CAL $(\mathrm{p}=0.31)$. This finding is similar to that of a study conducted by Bullon et al. [21] showing that there was no difference in CAL with regard to serum osteocalcin levels but suggesting that CAL was correlated with gingival crevicular fluid (GCF) and saliva osteocalcin levels. Osteocalcin is produced by osteoblasts and secreted directly into the blood in small amounts. Serum osteocalcin may not be a determinant of the periodontal status, as opposed to the osteocalcin in GCF and saliva because the serum levels reflect the bone turnover condition in whole bone.

The limitation of this study is that the no-risk, osteopenia risk, and osteoporosis risk groups of subjects were not equal in size. Subjects with a high osteoporosis risk were only a quarter of the total sample. However, this proportion was similar to the distribution of the osteoporosis prevalence in Indonesia. To get a better result, the author suggests a larger sample size in future research.

\section{CONCLUSION}

The results of this study showed no correlation of serum osteocalcin levels and periodontal attachment loss with osteoporosis risk in postmenopausal women, but serum osteocalcin levels were higher in the osteoporosis group than in the normal and osteopenia groups.
There is a positive correlation between periodontal attachment loss and osteoporosis high-risk status.

\section{ACKNOWLEDGMENTS}

This research was sponsored by a Grant for International Publication and indexed for a Student Final Project (Hibah PITTA 2017) from the Directorate of Research and Community Engagement of Universitas Indonesia, Jakarta, Indonesia.

The publication of this manuscript is supported by Universitas Indonesia.

\section{REFERENCES}

1. Bernabei R, Martone AM, Ortolani E, Landi F, Marzetti E. Screening, diagnosis and treatment of osteoporosis: A brief review. Clin Cases Miner Bone Metab 2014;11:201-7.

2. Esfahanian V, Shamami MS, Shamami MS. Relationship between osteoporosis and periodontal disease: Review of the literature. J Dent (Tehran) 2012;9:256-64 .

3. Widjanarko AL, Wachjudi RG, Chaidir MR. Do we need to perform bilateral hip bone mineral density examination? Indones J Rheumatol 2016;8:6-9.

4. Bodduru R, Deshmukh K, Chintawa S, Nayyar S. Osteoporosis and periodontal disease: Association and mechanisms: An in-depth review. Int J Therap Appl 2016;32:11-9.

5. Haq MW, Tanwir F, Tabassum S, Nawaz M, Siddiqui MF. Association of periodontitis and systemic diseases. Int J Dent Oral Health 2015;1:1-7.

6. Guiglia R, Di Fede O, Lo Russo L, Sprini D, Rini GB, Campisi G. Osteoporosis, jawbones and periodontal disease. Med Oral Patol Oral Cir Bucal 2013;18:e93-9.

7. Pepelassi E, Nicopoulou-Karayianni K, Archontopoulou AD, Mitsea A, Kavadella A, Tsiklakis K, et al. The relationship between osteoporosis and periodontitis in women aged 45-70 years. Oral Dis 2012;18:353-9.

8. Marques MR, Silva MA, Barros SP. Periodontal disease and osteoporosis association and mechanisms: A review of the literature. Braz J Oral Sci 2003;2:137-40.

9. Dodd DZ, Rowe DJ. The relationship between postmenopausal osteoporosis and periodontal disease. J Dent Hyg 2013;87:336-44.

10. Zanatta LC, Boguszewski CL, Borba VZ, Kulak CA. Osteocalcin, energy and glucose metabolism. Arq Bras Endocrinol Metabol 2014;58:444-51.

11. Hienz SA, Paliwal S, Ivanovski S, Cells B. Mechanisms of bone resorption in periodontitis author mechanisms of bone resorption in periodontitis. J Immunol Res 2015;2015:1-10.

12. Ali MR, Zaidan TF, Gorial FI. Validity of osteocalcin and alkaline phosphatase biomarkers in postmenopausal women with low bone mineral density. J Chemist and Mater Res 2014;6:13-9.

13. Singh S, Kumar D, Lal AK. Serum osteocalcin as a diagnostic biomarker for primary osteoporosis in women. J Clin Diagn Res 2015;9:4-7.

14. Kusdhany LS, Rahardjo TB, Sutrisna B. The development of mandibular bone density index to detect mandibular osteoporosis of postmenopausal women. Int J Oral Heath 2005;2:21-5.

15. Khanna V, Gupta P, Khanna R, Khanna HC. Postmenopausal osteoporosis. JOJ Case Stud 2017;1:1-5.

16. Juluri R, Prashanth E, Gopalakrishnan D, Kathariya R, Devanoorkar A, Viswanathan $\mathrm{V}$, et al. Association of postmenopausal osteoporosis and periodontal disease: A double-blind case-control study. J Int Oral Health 2015;7:119-23.

17. Bolarin DM, Ekpe EL, Saidu K, Eyam ES. A glance at biomarkers of bone metabolism. Pak J Nutr 2016;15:499-504.

18. Ram VS, Parthiban, Sudhakar U, Mithradas N, Prabhakar R. Bonebiomarkers in periodontal disease: A review article. J Clin Diagn Res 2015;9:ZE07-10.

19. Becerik S, Afacan B, Oztürk VÖ, Atmaca H, Emingil G. Gingival crevicular fluid calprotectin, osteocalcin and cross-linked N-terminal telopeptid levels in health and different periodontal diseases. Dis Markers 2011;31:343-52

20. Masulili SL, Tadjoedin FM, Masulili C, Indrasari M, Kusdhany LS. Periodontal attachment loss and bone mineral density. J Int Dent Med Res 2016;9:233-6.

21. Bullon P, Goberna B, Guerrero JM, Segura JJ, Perez-Cano R, Martinez-Sahuquillo A. Serum, saliva, and gingival crevicular fluid osteocalcin: Their relation to periodontal status and bone mineral density in postmenopausal women. J Periodontol 2005;76:513-9. 